\title{
COMPETÊNCIAS CURRICULARES PARA O LETRAMENTO CIENTÍFICO EM SAÚDE: POTENCIALIDADES E LIMITAÇÕES EM UMA INSTITUIÇÃO FEDERAL DE EDUCAÇÃO PROFISSIONAL
}

\section{COMPETENCIAS CURRICULARES PARA LA LITERACIA CIENTÍFICA EN SALUD: POTENCIALES Y LIMITACIONES EN UMA ESCUELA DE EDUCACIÓN \\ PROFESIONAL}

\begin{abstract}
LEARNING COMPETENCES FOR SCIENTIFIC LITERACY IN HEALTH: POTENTIALS AND LIMITATIONS IN AN EDUCATION IN PROFESSIONAL $S C H O O L$
\end{abstract}

\author{
Sueli da Silva COSTA ${ }^{1}$ \\ Mariana de Senzi ZANCUL ${ }^{2}$
}

RESUMO: A pesquisa apresentada no presente trabalho teve como objetivo promover a análise acerca da inserção do letramento científico focado na a educação em saúde nos currículos dos cursos técnicos integrados ao Ensino Médio do Instituto Federal de Brasília (IFB), para coleta de dados utilizou-se análise documental e para a avaliação optou-se pela técnica de análise de conteúdo. Pesquisou-se as competências relativas ao Letramento científico de caráter prático em saúde presentes nas componentes de ciências da natureza nos dezessete planos pedagógicos dos cursos técnicos integrados ao Ensino Médio do IFB. Cinco planos de curso não apresentaram competências relativa ao LC para o ES. Os outros doze apresentaram competências que foram categorizadas da seguinte maneira: 1. Relação saúde/ambiente; 2. Estratégias de manutenção da saúde; 3. Avanços tecnológicos e saúde; 4. Saúde/Políticas e sociocultural. Verificou-se que a inclusão destas categorias de temas no currículo dos cursos técnicos integrados ao EM do IFB procura convergir para a formação cidadã através de estratégias de letramento que, primeiramente, são previstas no currículo dos cursos e depois materializadas no cotidiano escolar.

PALAVRAS-CHAVE: Letramento científico. Educação em saúde. Ensino médio integrado.

RESUMEN: La investigación que se presenta en este trabajo tiene como objetivo promover el análisis sobre la inserción de la literacidad cientifica $(L C)$ centrada en la educación en salud (ES) en los currículos de los cursos técnicos integrados a la enseñanza secundaria del Instituto Federal de Brasilia (IFB). Para la recopilación de datos, se utilizó el análisis documental y para la evaluación se optó por la técnica de análisis de contenido. Se investigó las competencias relacionadas con la literacidad científica de carácter práctico en salud presentes

\footnotetext{
${ }^{1}$ Universidade Estadual de Campinas (UNICAMP), Campinas - SP - Brasil. Pesquisadora Colaboradora do Laboratório de Genética Molecular Humana, do Centro de Biologia Molecular e Engenharia Genética (CBMEG). Pós-doutorado em Genética e Biologia Molecular (UNICAMP). ORCID: http://orcid.org/0000-0002-2007-0933. E-mail: sueli.biologia@gmail.com

${ }^{2}$ Universidade de Brasília (UnB), Brasília - DF - Brasil. Docente no Núcleo de Educação Científica (NECBio) do Instituto de Ciências Biológicas. Doutorado em Alimentos e Nutrição (UNESP). ORCID: https://orcid.org/0000-0002-0027-8761.E-mail: marianaib@unb.br
} 
en los componentes de ciencias de la naturaleza en los diecisiete planes pedagógicos de los cursos técnicos integrados a la enseñanza secundaria del IFB. Cinco planes de curso no presentaban competencias relacionadas con la LC para la ES. Las otras doce presentaron competencias que fueron categorizadas de la siguiente manera: 1. Relación salud/ambiente; 2. Estrategias de mantenimiento de la salud; 3. Avances tecnológicos y salud; 4. Salud/Políticos y sociocultural. Se constató que la inclusión de estas categorías de temas en el currículo de los cursos técnicos integrados a la enseñanza secundaria del IFB busca converger hacia la formación ciudadana de los estudiantes, mediante estrategias de literacidad que, primeramente, son previstas en el currículo de los cursos y luego se materializan en el cotidiano escolar.

PALABRAS CLAVE: Literacia cientifica. Educación en salud. Escuela secundaria técnica.

ABSTRACT: This research aims to analyze the insertion of Scientific Literacy $(L C)$ in health education in Integrated Technical High School Curriculum of Federal Institute of Brasilia (IFB, Portuguese initials) using documentary analysis and content analysis technique to evaluate. The skills related to scientific literacy for health practices were researched in natural science subjects in seventeen pedagogical plans of integrated technical high school courses of IFB. Five course plans do not have competences related to scientific literacy for health education. The others contain competences that were categorized in that way: 1. Relation Health/Environment; 2. Health Maintenance Strategies; 3. Technological Progress and Health; 4. Health, policy, and culture. It was found that the insertion of these categories in integrated technical high school curriculum of IFB aims to promote a citizenship education through literacy strategies that are first provided in course planning and then materialized in daily school.

KEYWORDS: Scientific literacy. Health education. Integrated high school.

\section{Introdução}

O termo Letramento científico (LC) vem sendo amplamente utilizado entre os pesquisadores e professores atuantes na Educação em Ciências (CUNHA, 2017; AYALA, 2016; CERATI, 2014; FEINSTEIN, 2010; SANTOS, 2016; entre outros). Muitas das legislações relativas à formação do currículo escolar, especialmente no que diz respeito aos conhecimentos das ciências naturais, vêm sendo construídas com o foco em processos de ensino e aprendizagem que promovam um nível de domínio do conhecimento científico que possa ser caracterizado como letramento. Um exemplo recente é a publicação da Base Nacional Comum Curricular para a Educação Básica (BRASIL, 2017).

O processo de letramento científico torna-se ainda mais importante quando se lida com temas sensíveis à população, como a saúde individual e coletiva, por exemplo. Esforços no sentido de promover estratégias de ensino com foco no letramento científico são importantes especialmente no contexto atual que, com o advento das redes sociais, é caracterizado pelo 
incremento do que se convencionou denominar como fake news. Este tipo de informação exige grande capacidade de análise da realidade e de contextualização do que se lê e ouve com aquilo que é de fato realidade. Este é um problema global, mas especialmente grave nas questões relativas à saúde, uma vez que constrói relutâncias frente a eficiência e lisura do trabalho científico, especialmente das ciências médicas (MONARI; BERTOLLI FILHO, 2019).

A despeito deste importante processo, os debates sobre o conceito de letramento científico vêm acontecendo em torno de um termo ainda em construção. A utilização do conceito de Letramento científico ocorreu pela primeira vez na década de 1950 e passa por modificações de entendimento desde lá, não havendo ainda uma definição específica, nem consenso entre os estudiosos sobre o tema. De acordo com Ogunkola (2013), embora este consenso não esteja construído ainda, algumas dimensões são basilares para o entendimento do que é o letramento científico. Na primeira dimensão, denominada letramento nominal, o educando seria capaz de identificar termos e questões científicas, mas representaria tópicos, problemas, informações, conhecimentos ou compreensões acerca da ciência de forma incorreta. Além disto, surgem neste nível de letramento, representação de equívocos relacionados a conceitos e processos científicos. Geralmente, a pessoa com este nível de letramento fornece explicações insuficientes e inadequadas de fenômenos científicos. Considera-se, assim, que o educando faz uma utilização ingênua da ciência. O segundo nível, denominado letramento funcional, seria aquele onde o estudante é descrito como alguém capaz de utilizar o vocabulário científico de maneira adequada, definindo os termos científicos corretamente e memorizando palavras técnicas, ou seja, aqui reside o domínio da alfabetização científica, porém há dificuldade de contextualizá-las. Já no letramento científico Conceitual e Procedimental, o educando compreende esquemas conceituais da ciência, os processos da produção científica e as relações entre as partes de uma disciplina científica. No último nível, denominado Letramento científico Multidimencional, o indivíduo é capaz de compreender as qualidades da ciência e as diferenças de outros saberes e conhecimentos, reconhecendo sua história e a natureza das disciplinas científicas, além de ser capaz de entender a ciência em seus contextos sociais.

Além de Ogunkola, Shamos (1995) apresenta uma outra modalidade de análise dos estágios do LC. O autor afirma que o Letramento científico é a capacidade de compreender conhecimentos da ciência, o modo de pensar científico, suas estratégias de investigação e análise e a maneira como são construídos os modelos científicos. Para Shamos, o LC consiste, portanto, no domínio da linguagem científica, muito embora isto não se refira apenas ao domínio do vocabulário, mas, principalmente, de sua utilização para fins sociais. 
No contexto, o letramento científico poderia ser classificado em 3 categorias: a. Letramento científico de Ordem Cívica; b. Letramento científico de Ordem Cultural e c. Letramento científico de Ordem Prática (SHEN, 1975), sendo a terceira categoria a que levaria à formação cidadã dos estudantes e sobre a qual a presente pesquisa foi desenvolvida. Aqui também se pretendeu realizar uma análise curricular, deste modo, torna-se imprescindível discutir o conceito de currículo a que esta análise está ligada, uma vez que o currículo está intimamente conectado à concepção de homem e de sociedade que se pretende construir a partir dos processos de ensino e aprendizagem oriundos do modelo formativo.

Neste sentido, é importante levantar inicialmente que desde a antiguidade o homem se utiliza, em processos educativos, de conjuntos disciplinares organizados e atividades orientadas de maneira sistemática. No entanto, a palavra curriculum, no âmbito da educação apenas foi inserida em períodos mais recentes e surge em conjunto com a separação dos estudantes em classes (HAMILTON, 1992) e com o processo de compartimentalização do saber, muito presente na ciência Galileana, que se caracteriza pelo entendimento de que a ciência é um processo autônomo e que se encerra em si mesmo e que desconsidera os sujeitos envolvidos criando, a partir deste processo, categorias inequívocas (CASTORIADIS, 1997).

Do ponto de vista da etimologia do termo, a palavra currículo está associada ao termo do latim curriculu, que faz referência a uma pista de corrida. Silva (1999) reanalisando o termo em seus estudos aponta que, nesta corrida, acabamos por nos tornar o que somos. Os estudiosos de currículo que atuam na perspectiva crítica entendem que, neste constructo, estão envolvidas inúmeras tensões relativas as questões de poder em seu processo de construção e execução das estruturas curriculares (FREIRE, 1975; 2000; CORTELLA, 1998; CURY, 2000; MÉSZÁROS, 2005). Outros apontam suas contribuições especificamente sobre a análise das estruturas curriculares (APPLE, 2006; GIROUX, 1987; SACRISTÁN, 1998; 1999; 2002).

Sacristán (1999) afirma que “o currículo vem a ser uma espécie de texto cuja pretensão é a reprodução de uma forma de compreender a realidade e os processos de produção social aos quais ele deve servir". No entanto, Sacristán defende que entre os contextos de formulação e o de realização, há a intervenção de diversos agentes. Neste sentido, a escola e os professores não ensinam cultura ou conhecimento em abstrato, mas reconstruções deles, inscritos dentro de instituições e práticas cotidianas (SACRISTAN, 1999).

No que se refere ao currículo integrado desenvolvido no âmbitos dos cursos de formação profissional, Ramos (2009) aponta que para que o processo de integração entre a formação técnica e a básica (dada nos ensinos médios tradicionais) seja plenamente realizado, é necessário que três dimensões principais e complementares sejam satisfeitas: a integração entre 
as dimensões da formação humana (ciência, trabalho e cultura); a integração entre as formações do ensino médio e a formação técnica para a atuação profissional; e a integração das práticas docentes, no sentido da interdisciplinaridade e do trabalho conjunto.

Como parte dos currículos da educação de crianças e adolescentes no Brasil e ao redor de todo o mundo, inclusive na educação profissional, a Educação em Saúde é construída a partir de diversas concepções advindas da saúde e da educação e está em constante processo de construção/reconstrução. A Educação em Saúde vem recebendo muitas contribuições políticas e filosóficas ao longo do tempo, constituindo-se como processo de intervenção/interação sobre o indivíduo ou sobre um grupo de indivíduos, a fim de produzir neles os pré-requisitos para o desenvolvimento de escolhas que garantam a aquisição e manutenção da saúde. À despeito de sua importância, a Educação em Saúde vem sendo desenvolvida, em muitos casos, numa perspectiva prescritiva, biologicista e higienista, na qual se desconsidera a complexidade da ação humana e as possiblidades de reflexão, retroalimentação características das sociedades (VENTURI; MOHR, 2017).

Grande parte das ações de Educação em Saúde está associada ao debate de temas como o uso de drogas e medicamentos, sexualidade e doenças sexualmente transmissíveis, usos e resistências à vacinação e, ainda, alimentação e relações entre saúde e meio ambiente. Do ponto de vista teórico, a Educação em Saúde é compreendida como o processo educativo de construção de conhecimentos para a apropriação do tema pela população como um todo, buscando o aumento da autonomia dos indivíduos no autocuidado (FALKENBERG, 2014). Por fim, a Educação em Saúde consiste, ainda, na produção e sistematização de conhecimentos relativos à formação e ao desenvolvimento para a atuação em saúde, envolvendo práticas de ensino, diretrizes didáticas e orientação curricular (BRASIL, 2018).

Para que a Educação em Saúde atinja os fins ora apresentados, é recomendável que ela faça parte da rotina escolar, inserindo-se e contextualizando-se a este ambiente, pois assim os conhecimentos necessários a uma forma de vida mais saudável serão desenvolvidos de maneira permanente e perdurarão tanto no espaço escolar, quanto fora dele (MAINARD, 2010).

Diante das ideias aqui apresentadas, esta pesquisa teve como objetivo analisar a possível inserção de competências voltadas para o letramento científico em saúde nos currículos dos cursos técnicos integrados ao Ensino Médio do Instituto Federal de Brasília (IFB), Brasil. 


\section{Metodologia}

Nesta investigação, optou-se por estratégias de pesquisa qualitativa. De acordo com Yin (2016), a pesquisa qualitativa é um campo de investigação que se caracteriza, principalmente, por analisar processos relativos à existência dos indivíduos como seres sociais, captando, através de técnicas adequadas, as visões, concepções e perspectivas das diversas facetas da vida humana. Nos processos investigativos associados à pesquisa qualitativa são levados em consideração os contextos nos quais os investigados estão inseridos, além dos fenômenos que possam, porventura, influenciar suas escolhas, o que é o caso da formação de currículos escolares.

A pesquisa qualitativa também pode ser compreendida como aquela que considera as interrelações entre os indivíduos participantes da investigação e a realidade na qual eles estão inseridos, uma vez que eles estão indissociavelmente ligados, sendo difícil separar o mundo (objetivo) do sujeito (subjetivo), relação esta que não pode ser mensurada numericamente. Neste tipo de pesquisa, a interpretação dos fenômenos e a atribuição de significados aos mesmos são o objetivo básico da investigação (KAUARK et al., 2010).

Diante do exposto até aqui e partindo da compreensão de Currículo como artefato histórico e considerando as características da Educação em Saúde e do Letramento científico, optou-se, na coleta de dados, pela análise documental (KAUARK; MANHÃES; MEDEIROS, 2010). Para a avaliação dos dados coletados optou-se pela técnica de análise de conteúdo (BARDIN, 2011).

A Análise de Conteúdo foi sistematizada por Bardin em seu livro Analyse de Contenu em 1977. De acordo com Bardin (2011), a expressão análise de conteúdo diz respeito a:

Um conjunto de técnicas de análise das comunicações visando a obter, por procedimentos sistemáticos e objetivos de descrição do conteúdo das mensagens, indicadores (quantitativos ou não) que permitam a inferência de conhecimentos relativos às condições de produção/recepção (variáveis inferidas) destas mensagens (BARDIN, 2011, p. 47).

Esta estratégia de análise busca compreender as características, estruturas ou modelos inseridos nas mensagens produzidas nas mais diversas formas de comunicação. A análise de conteúdo, aprimorada por Bardin, é composta por três etapas principais: 1. Pré-análise; 2. Exploração do material; e 3. Tratamento dos resultados - a inferência e a interpretação (CÂMARA, 2013).

A pré-análise consiste na leitura do material obtido na fase da coleta de dados. Ela é realizada, geralmente através de uma leitura flutuante de entrevistas ou relatos produzido pelo 
grupo analisado. Nesta leitura é necessária a observação de pontos de convergência nos discursos produzidos, pois estes serão utilizados na fase seguinte, da elaboração das categorias de análise (SILVA, 2005).

Na exploração do material são estabelecidas as unidades de codificação que resultarão em categorias, as quais devem ser associados aos discursos que as justificam. Podem ser escolhidas para o estabelecimento destas categorias, palavras, expressões e léxicos a partir dos quais os registros possam ser agrupados. Os dados devem ser organizados em tabelas que serão preenchidas com colunas onde constem as categorias elaboradas e os excertos dos discursos obtidos no período de coleta. A última categoria refere-se ao tratamento dos resultados - a inferência e interpretação. Nesta etapa, com as categorias estabelecidas e discursos vinculados, o pesquisador passa a elaborar as proposições e conceitos que se obteve nas etapas anteriores e, finalmente a interpretação dos dados, onde alicerçado no referencial teórico previamente escolhido, o investigador interpreta os dados fazendo inferências, comparando com outros estudos em mesmo sentido, movimento a partir do qual o pesquisador formará suas conclusões acerca do fenômeno estudado (CÂMARA, 2013).

Com base em tais ideias, foi composta uma amostra um total de quinze planos pedagógicos dos cursos técnicos integrados à formação profissional do IFB. Neles, foram analisados os currículos das disciplinas de Ciências da Natureza, nas quais esperava-se identificar a presença de competências direcionadas para a formação do letramento científico para os temas relativos à saúde.

\section{Resultados e discussão}

Nesta pesquisa analisou-se os dezessete planos pedagógicos dos Cursos Técnicos Integrados ao Ensino Médio do Instituto Federal de Brasília afim de identificar possíveis competências relativas ao letramento científico, de caráter prático, em saúde nos componentes curriculares de Ciências da Natureza.

Dos planos avaliados, cinco não apresentaram competências relativas ao à Educação em Saúde. Os outros 12 apresentaram tais competências que foram categorizadas da seguinte maneira: 
Tabela 1 - Categorização da Análise de Conteúdo das competências em Saúde dos Projetos Pedagógicos dos Cursos Técnicos Integrados ao Ensino Médio do IFB

\begin{tabular}{|c|c|c|}
\hline Categoria & Subcategoria & Indicador \\
\hline \multirow{2}{*}{$\begin{array}{l}\text { Competência } \\
\text { Curricular em } \\
\text { Saúde }\end{array}$} & $\begin{array}{c}\text { Relação } \\
\text { saúde/ambiente }\end{array}$ & $\begin{array}{l}\text { PC.6: "Compreender a importância do controle e } \\
\text { monitoramento do meio ambiente, por meio de técnicas } \\
\text { adequadas, de modo a preservar os recursos naturais e garantir } \\
\text { saúde e bem estar da população" } \\
\text { PC.8: "Compreender interações entre organismos e ambiente, } \\
\text { em particular aquelas relacionadas à saúde humana, } \\
\text { relacionando conhecimentos científicos, aspectos culturais e } \\
\text { características individuais" } \\
\text { PC.11: "Identificar os riscos referentes aos fatores ambientais } \\
\text { condicionantes e determinantes das doenças e outros agravos à } \\
\text { saúde"; "Estimular a interação entre saúde, meio ambiente e } \\
\text { desenvolvimento, visando ao fortalecimento da participação da } \\
\text { população na promoção da saúde e qualidade de vida." } \\
\text { PC.3: "Reconhecer que as condições de alimentação, de } \\
\text { educação e do meio ambiente, dentre outros, são fatores } \\
\text { determinantes da saúde individual e coletiva." } \\
\text { PC.12: "Reconhecer que as condições de alimentação, de } \\
\text { educação e do meio ambiente, dentre outros, são fatores } \\
\text { determinantes da saúde individual e coletiva." }\end{array}$ \\
\hline & $\begin{array}{c}\text { Estratégias de } \\
\text { manutenção da saúde }\end{array}$ & $\begin{array}{l}\text { PC.1: "Compreender meios e atitudes para promoção e } \\
\text { preservação da saúde."; "Compreender os aspectos etiológicos } \\
\text { sobre as doenças causadas por infecções e aspectos de saúde } \\
\text { coletiva"; "Reconhecer os vários tipos de drogas e os malefícios } \\
\text { causados à saúde pela sua utilização, relacionando os efeitos } \\
\text { sofridos pelo organismo humano." } \\
\text { PC.2: "Reconhecer os vários tipos de drogas e os malefícios } \\
\text { causados à saúde pela sua utilização, relacionando os efeitos } \\
\text { sofridos pelo organismo humano." } \\
\text { PC.3: "Fomentar diálogos e ações interventivas sobre a saúde } \\
\text { pública e a prevenção de doenças relacionadas ao ambiente de } \\
\text { trabalho." } \\
\text { PC.4: "Refletir sobre o funcionamento do organismo humano, } \\
\text { de forma a adotar uma postura autônoma de seleção de } \\
\text { atividades e procedimentos na manutenção e aquisição da } \\
\text { saúde." } \\
\text { PC.5: Relacionar o saneamento básico com a saúde humana } \\
\text { nas diversas regiões brasileiras. } \\
\text { PC.9: "Valorizar os conhecimentos sobre a estrutura e o } \\
\text { funcionamento dos sistemas de órgãos do corpo humano, } \\
\text { reconhecendo-os como necessários tanto para a identificação de } \\
\text { eventuais distúrbios orgânicos como para os cuidados com a } \\
\text { manutenção da própria saúde"; "Conhecer suplementos } \\
\text { alimentares, esteroides anabólicos-androgênicos e aceleradores } \\
\text { metabólicos, seu funcionamento e os efeitos à saúde" } \\
\text { PC.8: "Avaliar propostas de alcance individual ou coletivo, } \\
\text { identificando aquelas que visam à preservação e a } \\
\text { implementação da saúde individual, coletiva ou do ambiente" }\end{array}$ \\
\hline
\end{tabular}




\begin{tabular}{|l|l|l|}
\hline \multirow{1}{*}{} & $\begin{array}{l}\text { PC.10: “Entender a importância dos nutrientes na formação das } \\
\text { células e na manutenção da saúde" }\end{array}$ \\
\cline { 2 - 3 } & $\begin{array}{l}\text { Avanços tecnológicos } \\
\text { e saúde }\end{array}$ & $\begin{array}{l}\text { PC.5: "Reconhecer os benefícios da biotecnologia à saúde e à } \\
\text { produção de alimentos" }\end{array}$ \\
\hline Política/Sociocultural & $\begin{array}{l}\text { PC.1: "Construir a noção de saúde levando em conta os } \\
\text { condicionantes biológicos como sexo, idade, fatores genéticos } \\
\text { e os condicionantes sociais, econômicos, ambientais e culturais } \\
\text { como nível de renda, escolaridade, estilos de vida, estado } \\
\text { nutricional, possibilidade de lazer, qualidade do transporte, } \\
\text { condições de saneamento." } \\
\text { PC.6: "Identificar, analisar e discutir vulnerabilidades } \\
\text { vinculadas às vivências e aos desafios contemporâneos aos } \\
\text { quais as juventudes estão expostas, considerando os aspectos } \\
\text { físico, psicoemocional e social, a fim de desenvolver e divulgar } \\
\text { ações de prevenção e de promoção da saúde e do bem-estar."; } \\
\text { "avaliar e/ou promover ações que contribuam para a melhoria } \\
\text { na qualidade de vida e nas condições de saúde da população" } \\
\text { PC.7: "Adotar atitudes que promovam a ampliação permanente } \\
\text { da qualidade de vida, reconhecendo a saúde como aspecto } \\
\text { humano que envolve questões biológicas, políticas, econômicas } \\
\text { e socioculturais"; "Refletir sobre o papel das estratégias de } \\
\text { higiene para a manutenção do estado geral de saúde" }\end{array}$ \\
\hline
\end{tabular}

Legenda: PC- Plano de Curso

Fonte: Elaborado pelas autoras

Na categoria 1- Relação saúde/ambiente - podem ser inseridas as competências que buscam construir com os estudantes a capacidade de relacionar problemas e saúde e a relação saúde/doença com alterações no equilíbrio ambiental. Estudos anteriores apontaram a necessidade de um olhar holístico sobre as relações entre saúde e meio ambiente, pois há entre elas uma inter-relação oriunda da impossibilidade de separação do homem do ambiente natural (LEFF, 2001) (BRAGA et al., 2007) (NEGRETE et al., 2010).

Neste sentido, em estudo realizado nos currículos dos cursos de graduação das áreas de saúde, Souza e Andrade (2014) avaliaram que a incorporação das questões do meio ambiente e suas relações com as políticas de saúde, além da análise dos objetivos da saúde ambiental mostraram-se relevantes na formação dos futuros profissionais de saúde, principalmente quando se pensa na necessidade de fomentar nos estudantes a percepção de que sua existência individual é parte de um ambiente natural. Neste processo é possível que, através desta formação, o educando seja capaz de apontar as inter-relações entre homem e natureza, as relações de reciprocidade entre os elementos componentes do mundo natural, incluída a espécie humana, colocando-a como elemento constituinte do ambiente e não apartada dele. 
Na categoria 2- Estratégias de manutenção da saúde - estão inseridas as competências que têm como característica principal a intenção de promoção/manutenção da saúde. $\mathrm{Na}$ maioria das vezes estas competências estão relacionadas a um viés biologicista da educação em saúde e remetem as referências deste tipo de ação educativa no Brasil. Entende-se como viés biologicista, uma abordagem da educação em saúde voltada exclusivamente para questões biológicas da relação saúde/doença. Uma ideia de que apenas os conhecimentos acerca de como funcionam as espécies e suas relações seria suficiente para o estabelecimento de um estado de saúde. Embora a ES tenha mudado, esta ainda é a principal abordagem de educação em saúde desenvolvida dos espaços escolares, principalmente nas componentes curriculares de ciências da natureza (SILVA; TEIXEIRA; FERREIRA, 2012).

Monteiro e Bizzo (2015), em pesquisa que analisava o histórico da inserção dos temas relativos à manutenção da saúde nos currículos escolares, apontam que, apesar de uma presença incipiente da educação em saúde nos documentos oficiais da educação brasileira ao longo dos últimos 40 anos, são notórios os avanços e alterações na compreensão do conceito sobre o tema nas políticas públicas educacionais. Os autores sugerem ainda que os documentos curriculares abandonem o caráter prescritivo da ES, propiciando aos estudantes a valorização e utilização das características peculiares das sociedades onde estão inseridos, medida que apenas pode ser adotada quando o currículo é desenhado com base na realidade social onde será executado.

Já a categoria 3- Avanços tecnológicos e saúde - compreende as competências que possuem, entre as analisadas, maior potencial do ponto de vista do letramento científico. A valorização dos resultados da produção científica e tecnologia tiveram um incremento significativo no século XX, de modo de modo que hoje é impensável o trabalho em ES sem levar em consideração as tecnologias, muitas delas de caráter inovador (SOUZA, 2016).

Martins (2019), refletindo a respeito das possibilidades de articulação da educação em saúde, aponta que uma das formas efetivas de promover uma ES sustentável e significativa é sua integração ao estudo dos avanços científicos e tecnológicos. A autora afirma ainda que uma execução mais integrada e crítica das questões de saúde nos currículos escolares acontece quando esta é realizada com a inclusão de discussões sobre os avanços tecnológicos e, com a incorporação dos determinantes sociais destes avanços, o que pode ser obtido quando a ES é abordado na perspectiva da Educação para a Ciência-Tecnologia-Sociedade (CTS).

A categoria 4- Saúde e Política/Sociocultural - refere-se à contextualização da ES e está baseada na concepção de educação como um processo que envolve ação-reflexão-ação. Competências educacionais com esta característica evidenciam a necessidade de uma formação educativa que vise uma ação concreta, cultural, política e social com base no apontamento de 
situações conflitantes do cotidiano, a percepção de contradições no contexto social e ações para a sua superação (FREIRE, 1987).

Nespolo e colaboradores (2014) analisaram o trabalho em educação em saúde associada à educação popular através dos Pontos de Cultura. Deste processo de ação e reflexão, os autores levantaram que a associação entre ES e as questões sociais e culturais, nestes Pontos, constituise como uma estratégia de amplificação do trabalho dialógico, onde os envolvidos são agentes de mobilização das questões de saúde. Neste processo há a valorização dos saberes, experiências e emoções. Por meio deste modelo de educação em saúde é possível potencializar o envolvimento popular em ações voltadas à promoção da saúde individual e coletiva, o que incrementa a participação coletiva e os laços de solidariedade na comunidade (NESPOLO et al., 2014).

Diante dos dados encontrados nos currículos dos cursos Técnicos Integrados do IFB, verificou-se que a inclusão destas categorias de temas na formação dos estudantes caracterizase como uma forma de estímulo à formação cidadã, através de estratégias de letramento que primeiramente são previstos no currículo dos cursos, materializados no cotidiano escolar e, espera-se, materializados nas escolhas mais saudáveis no cotidiano dos estudantes.

\section{Considerações finais}

A análise dos currículos dos Cursos Técnicos Integrados do Instituto Federal de Brasília, no que se refere à inclusão de temas que têm potencial para o desenvolvimento do Letramento científico em saúde, reflete uma preocupação e uma tentativa de enfatizar a importância do tema para a formação de estudantes adolescentes que, muitas vezes, necessitam tomar decisões acerca da utilização de drogas, dos comportamentos sexuais e da saúde psicológica, por exemplo.

Deste processo, verifica-se que, em consonância com as orientações curriculares oficiais do Brasil, há a inserção do tema na maioria dos cursos da instituição havendo, no entanto, a necessidade de acréscimo deste debate em cinco planos de curso.

Dos planos onde já há a previsão do debate sobre saúde, alguns apresentam maior potencial do letramento prático, que é necessário para o desenvolvimento de uma ES mais efetiva e com resultados melhores. Nestes planos a educação em saúde vêm relacionada a reflexões socioculturais, diminuindo o caráter biologicista e higienista que muitas vezes encontramos na Educação em Saúde. 
Acredita-se, a partir das informações obtidas neste estudo, que seja necessária a realização de pesquisas destinadas a verificar os modos como estas informações curriculares são materializadas em sala de aula, ou seja, as práticas realmente desenvolvidas no cotidiano de estudantes e professores, além das contribuições efetivas que este debate traz para a promoção da saúde e da vida dos estudantes.

\section{REFERÊNCIAS}

ALVES, V. S. Um modelo de educação em saúde para o programa saúde da família: pela integralidade da atenção e a reorientação do modelo assistencial. Interface - Comunicação, Saúde e Educação, Botucatu, v. 9, p. 39-52, fev. 2005.

APPLE, M. (Org). Ideologia e currículo. Porto Alegre: Artes Médicas, 2006.

AYALA, F. J. Introductory essay: the case for scientific literacy. Paris: UNESCO, 1996.

BARDIN, L. Análise de conteúdo. São Paulo: Edições 70, 2011.

BRAGA, A. L. et al. Association between air pollution and respiratory and cardiovascular diseases in Itabira. Cadernos de Saúde Pública, v. 23, supl. 4, p. 570-578, 2007.

BRASIL. Base Nacional Comum Curricular. Brasília, DF: MEC, 2017. Disponível em: http://basenacionalcomum.mec.gov.br/images/BNC C_20dez_site.pdf. Acesso em: 22 de dezembro de 2017.

CASTORIADIS, C. Encruzilhadas do labirinto. Rio de Janeiro: Paz \& Terra, 1987.

CERATI, T. M. Educação em jardins botânicos na perspectiva de alfabetização científica: análise de uma exposição e público. Orientador: Martha Marandino. 2014. 254 f. Tese (Doutorado em Educação) - Universidade de São Paulo, São Paulo, 2014.

CORTELLA, M. S. A escola e o conhecimento: fundamentos epistemológicos e políticos. São Paulo: Cortez/Instituto Paulo Freire, 1998.

CUNHA, R. B. Alfabetização científica ou letramento científico? Interesses envolvidos nas interpretações da noção de scientific literacy. Rev. Bras. Educ., Rio de Janeiro, v. 22, n. 68, p. 169-186, 2017.

CURY, C. J. Educação e contradição. São Paulo: Cortez, 2000.

FALKENBERG, M.; MENDES, T. P. L.; MORAES, E. P.; SOUZA, E. M. Educação em saúde e educação na saúde: conceitos e implicações para a saúde coletiva. Ciênc. saúde coletiva, Rio de Janeiro, v. 19, n. 3, p.847-852, 2014.

FEINSTEIN, N. Salvaging science literacy. Science Education, Hoboken: John Wiley \& Sons, v. 95, n. 1, p. 168-185, 2010.

FREIRE, P. Pedagogia do oprimido. Rio de Janeiro: Paz e Terra, 1975. 
FREIRE, P. Pedagogia da indignação. São Paulo, UNESP, 2000.

FREIRE, P. Pedagogia do oprimido. 17. ed. Rio de janeiro: Paz e terra, 1987.

GIROUX, H. A escola crítica e política cultural. São Paulo: Cortez e A.A., 1987.

HAMILTON, D. Sobre as origens do termo classe e curriculum. Teoria e Educação, Porto Alegre, v. 6, 1992.

KAUARK, F.; MANHÃES, F.; MEDEIROS, C. Metodologia de pesquisa: um guia prático. Itabuna: Via Litterarum, 2010.

LEFF, E. Saber ambiental: sustentabilidade, racionalidade, complexidade. Petrópolis: Ed. Vozes, 2001.

MAINARD, N. Educação em saúde: problema ou solução? Orientador: Isabel Maria Teixeira Bicudo Pereira. 2010. 135 f. Tese (Doutorado em Saúde Pública) - Universidade de São Paulo. São Paulo, 2010.

MARTINS, I. Educação em Ciências e Educação em Saúde: breves apontamentos sobre histórias, práticas e possibilidades de articulação. Revista Ciência e Educação, Bauru, v. 25, n. 2, p. 269-275, 2019.

MÉSZÁROS, I. A educação para além do capital. São Paulo: Ed. Boitempo, 2005.

MONTEIRO, P. H. N; BIZZO, N. A saúde na escola: análise dos documentos de referência nos quarenta anos de obrigatoriedade dos programas de saúde, 1971-2011. História, Ciências, Saúde - Manguinhos, Rio de Janeiro, v. 22, n. 2, p. 411-427, abr./jun. 2015.

MONARI, A. C. P.; BERTOLLI FILHO, C. Saúde sem fake news: estudo e caracterização das informações Falsas divulgadas no canal de informação e checagem de fake news do ministério da saúde. Revista Mídia e Cotidiano, Niterói, v. 13, n. 1, p. 160-186, mar. 2019.

NEGRETE, B. et al. Air pollution and hospital admissions of adults and elderly due to congestive heart failure in santo andré. Arquivos Brasileiros de Ciências da Saúde, Santo André, p. 50-62, 2010.

NESPOLO G. F. et al. Culture points: contributions to popular health education in the perspective of their coordinators. Interface, Botucatu, v. 18, supl. 2, p. 1187-1198, 2014.

OGUNKOLA, B. J. Scientific Literacy: Conceptual Overview, Importance and Strategies for Improvement. Journal of Educational and Social Research, p. 265-274, 2013.

SACRISTÁN, J. G. O currículo: uma reflexão sobre a prática. Porto Alegre: Ed. Artes Médicas, 1998.

SACRISTÁN, J. G. Poderes instáveis em educação. Porto Alegre: Ed. Artes Médicas, 1999. SACRISTÁN, J. G. Educar conviver na cultura global: as exigências da cidadania. Porto Alegre: Ed. Artes Médicas, 2002. 
SANTOS, W. L. P. Educação científica na perspectiva de letramento como prática social: funções, princípios e desafios. Revista Brasileira de Educação, Rio de Janeiro: ANPEd; Campinas: Autores Associados, v. 12, n. 36, p. 474-550, 2007.

SHAMOS, M. H. The myth of literacy. New Brunswick: Rutgers University Press, 1995.

SHEN, B. S. Science literacy. American Scientist, New York, n. 63, p. 265-268, 1975.

SILVA, J.; TEIXEIRA, M. L.; FERREIRA, M. A. Alimentação e saúde: sentidos atribuídos por adolescentes. Revista de Enfermagem Escola Anna Nery, Rio de Janeiro, v. 16, n. 1, p. 88-95, 2012.

SILVA, T. T. Documentos de identidade: uma introdução às teorias do currículo. Belo Horizonte: Autêntica, 1999.

SOUZA, L. E. Saúde, desenvolvimento e inovação: uma contribuição da teoria crítica da tecnologia ao debate. Cad. Saúde Pública, São Paulo, v. 32, supl. 2, p. S1-S10, 2016.

VENTURI, T.; MOHR, A. Aproximação pesquisa e prática docente: contribuições de um curso de formação de professores no tema Educação em Saúde. Ensenanza de Las Ciencias, ed. extraordinária, p. 443-448, 2017.

YIN, R. K. Pesquisa qualitativa do início ao fim. Porto Alegre: Penso, 2016.

\section{Como referenciar este artigo}

COSTA, S. S.; ZANCUL, M. S. Competências curriculares para o letramento científico em saúde: potencialidades e limitações em uma instituição federal de educação profissional. Temas em Educ. e Saúde, Araraquara, v. 16, n. 2, p. 607-620, jul./dez. 2020. e-ISSN 2526-3471. ISSN 1517-7947. DOI: https://doi.org/10.26673/tes.v16i2.14095

Submetido em: $25 / 04 / 2020$

Revisões requeridas: $19 / 06 / 2020$

Aprovado em: 28/07/2020

Publicado em: 27/08/2020 